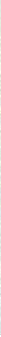

\title{
A construção civil sob o ponto de vista da avaliação da sustentabilidade do ciclo de vida - revisão
}

The civil construction under the life cycle sustainability assessment standpoint - review

La construcción civil bajo el punto de la evaluación de la sostenibilidad del ciclo de vida - revisión

Daniel Oliveira de Azevedo Sampaio Aluno de Graduação, UNESP, Brasil. daniel.oliveira.oas@hotmail.com

Camila Cassola Assunção Aluna de Pós-Graduação, UNESP, Brasil. camila.cassola.assuncao@gmail.com

Jorge Luís Akasaki

Professor Doutor, UNESP, Brasil. jorge.akasaki@gmail.com 


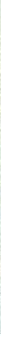

\title{
RESUMO
}

A Avaliação da Sustentabilidade do Ciclo de Vida (ASCV) é uma ferramenta que pode ser utilizada para avaliar a sustentabilidade da perspectiva do ciclo de vida, e vem sendo alvo crescente de estudos nos últimos anos. Esse artigo tem como objetivo apresentar essa metodologia desenvolvida recentemente, através de uma revisão da bibliografia, com foco no setor da construção civil, para obter as aplicações usuais, vantagens, limitações e desafios. A abordagem mais comum da ASCV é uma união de três métodos distintos, sem ponderação formal entre eles, relacionados com os pilares da sustentabilidade: Avaliação do Ciclo de Vida, Avaliação dos Custos do Ciclo de Vida e a Avaliação do Ciclo de Vida Social. Poucos artigos utilizando essa metodologia em sistemas e materiais envolvidos com a construção civil foram encontrados. Os principais desafios para a ampla aplicação da ASCV são a carência de harmonia entre os métodos que a compõe e a falta de interesse do público geral em incorporar a sustentabilidade em seus produtos e serviços, por temer o encarecimento e a queda na qualidade. Ainda é necessária a realização de mais estudos de caso, em diversos setores diferentes, para evidenciar as particularidades de cada um e aprimorar essa metodologia para sua ampla utilização, além da criação de uma norma voltada especificamente para essa avaliação, facilitando a execução e comparação de estudos.

PALAVRAS-CHAVE: Avaliação de Sustentabilidade do Ciclo de Vida (ASCV). Desenvolvimento sustentável. Construção civil.

\begin{abstract}
The Life Cycle Sustainability Assessment (LCSA) is a tool that can be used to assess sustainability from a life cycle perspective, and it has been the focus of studies in recent years. This article aims to present this recently developed methodology, through a literature review, focusing on the construction sector, to obtain its usual applications, advantages, limitations and challenges. LCSA's most common approach is a combination of three distinct unweighted methods, related to the three main pillars of sustainability: Life Cycle Assessment, Life Cycle Cost Assessment and Social Life Cycle Assessment. Few articles using this methodology in systems and materials involved with the civil construction were found. The main challenges for the broad application of LCSA are the absence of harmony between its methods and the general public's lack of interest in incorporating sustainability into theirs products and services, fearing an increase in the costs and a drop in quality. Further case studies in several sectors are still necessary to highlight the particularities of each one and to improve this methodology for its wide use, besides the development of a standard for this assessment, facilitating the execution and the comparison of studies.
\end{abstract}

KEYWORDS: Life Cycle Sustainability Assessment (LCSA). Sustainable development. Civil construction.

\section{RESUMEN}

La Evaluación de la Sostenibilidad del Ciclo de Vida (ESCV) es una herramienta que se puede utilizar para evaluar la sostenibilidad desde la perspectiva del ciclo de vida, y ha sido el foco de los estudios en los últimos años. Este artículo tiene como objetivo presentar esta metodología recientemente desarrollada, a través de una revisión de la literatura, centrada en el sector de la construcción civil, para obtener las aplicaciones habituales, ventajas, limitaciones y desafíos. El enfoque más común de ASCV es la unión de tres métodos distintos, no ponderados formalmente, relacionados con los pilares de la sostenibilidad: Evaluación del Ciclo de Vida, Evaluación del Costo del Ciclo de Vida y Evaluación del Ciclo de Vida Social. Se encontraron pocos artículos que utilizan esta metodología en sistemas y materiales relacionados con la construcción civil. Los principales desafíos para la aplicación general de ESCV son la falta de armonía entre sus métodos y la falta de interés del público en general por incorporar la sostenibilidad en sus productos y servicios, por temer el aumento de precio y la caída de la calidad. Aún son necesarios más estudios de caso en varios sectores diferentes para resaltar las particularidades de cada uno y mejorar esta metodología para su amplio uso, además de la creación de una normativa para esta evaluación, facilitando la ejecución y comparación de estudios.

PALABRAS CLAVE: Evaluación de la Sostenibilidad del Ciclo de Vida (ESCV). Desarrollo sostenible. Construcción civil. 


\section{INTRODUÇÃO}

A indústria da construção civil é extremamente significante para uma nação, já que influencia demasiadamente em aspectos ambientais, sociais e econômicos (YUSOF; IRANMANESH, 2017; MARCELINO-SADABA et al., 2017; SILVA et al., 2018). O setor consome globalmente cerca de $14 \%$ a $50 \%$ da matéria-prima extraída da natureza, $16 \%$ dos recursos hídricos, $40 \%$ de toda fonte de energia, gera de $40 \%$ a $70 \%$ dos resíduos sólidos e cerca de $10 \%$ das emissões de gases de efeito estufa na atmosfera, já que é responsável pelas atividades ligadas desde a extração de matéria prima até a descarte dos resíduos, incluindo todos os reparos e manutenções necessários durante a vida útil das construções (MEDEIROS, DURANTE, CALLEJAS, 2018; TAVARES, 2006; ROODMAN et al., 1995). No Brasil, segundo Teixeira e Carvalho (2005), o setor é composto quase que unicamente por pequenas e micro empresas, que empregam mão de obra de forma intensa, majoritariamente pouco qualificada. Além disso, a indústria construção, no geral, utiliza métodos construtivos tradicionais, que consomem matérias primas e fontes energéticas de maneira acelerada, gerando uma grande quantidade de resíduos; produz a maioria de seus insumos de forma local ou regional; e tem alto impacto sobre a economia, através do grande número de empregos e da carga tributária gerada pelos diversos empreendimentos (MATHEUS; BRAGANÇA, 2004).

Todos esses aspectos negativos, porém, podem ser vistos por uma ótica diferente, já que a indústria da construção tem um grande potencial de melhora, podendo transformar os pontos desfavoráveis em oportunidades de melhorias e inovações, buscando-se o desenvolvimento sustentável, ou seja, o desenvolvimento capaz de suprir as necessidades da sociedade atual sem comprometer as necessidades das gerações futuras (NAÇÕES UNIDAS, 1987). Seria interessante encontrar alternativas para o modelo atual, onde a matéria-prima extraída é transformada e em seguida descartada (AZEVEDO, 2015). As modificações e melhorias podem ocorrer tanto nos métodos construtivos, racionalizando as construções e otimizando o uso de insumos, reduzindo assim a quantidade de resíduos; ou então nos materiais e em novos compósitos que incorporem resíduos, por exemplo, podendo melhorar as propriedades dos materiais convencionais, diminuindo a quantidade de matéria prima despendida e propiciando um valor agregado ao resíduo que antes seria simplesmente descartado.

Figura 1: Pilares da sustentabilidade

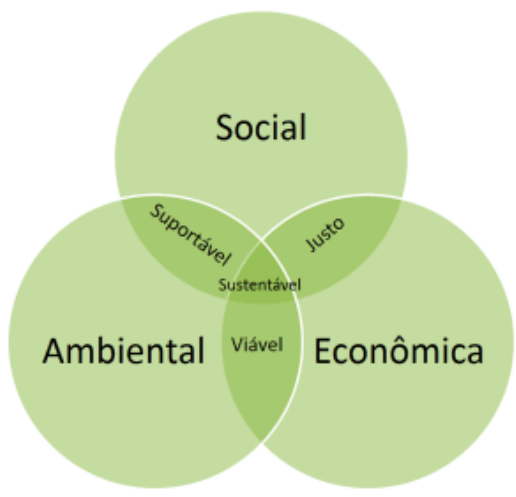

Fonte: LOBO, 2016. 


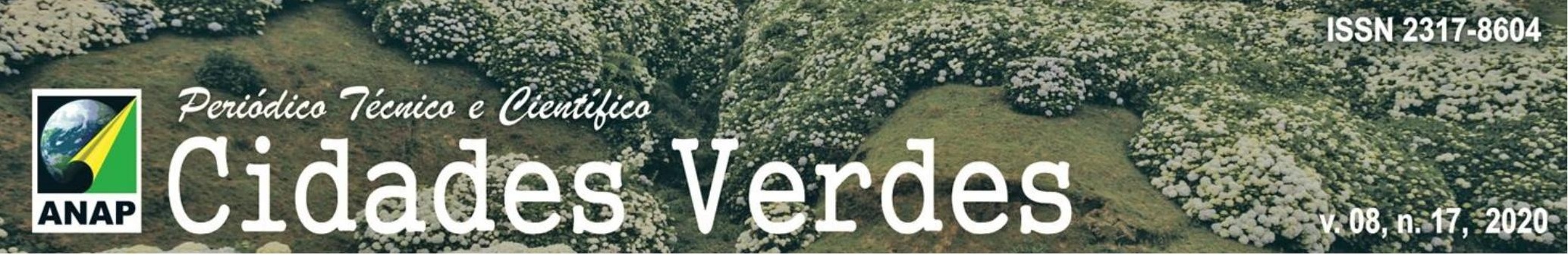

A sustentabilidade, de forma geral, possui três frentes principais, ilustradas na Figura 1. Porém, como foi destacado anteriormente, a grande maioria dos edifícios e construções não seguem um padrão voltado para a sustentabilidade, sendo normalmente empregados métodos e materiais convencionais. Segundo a Comissão Europeia (UE, 2004), o principal desafio a ser superado é a falta de interesse dos clientes e das construtoras, por acreditarem, de forma equivocada, que esse tipo de construção voltada para a sustentabilidade só traz mais custos e não possui o mesmo desempenho e confiabilidade a longo prazo.

Segundo Batlouni Neto (2007), a maneira mais efetiva de enfrentar as particularidades negativas da construção civil seria utilizar materiais que gerem menor volume de resíduos, utilizar insumos que possam ser reciclados com maior facilidade, ou então incorporar resíduos nas construções, através do desenvolvimento de novos compósitos, por exemplo. Um extenso número de pesquisas são realizadas anualmente sobra utilização de materiais alternativos, normalmente levando em conta apenas aspectos técnicos (no caso da construção civil, avaliando principalmente as propriedades mecânicas), e eventualmente avaliando aspectos econômicos ou ambientais. Porém, como foi discutido até então, não basta apenas incorporar esses resíduos sem realizar um estudo minucioso, já que, ao modificar a composição de um material convencional, não necessariamente o impacto ambiental, social e econômico será mitigado.

Os produtos da indústria da construção civil, sejam eles convencionais ou então desenvolvidos através de técnicas e materiais alternativos, precisam ser avaliados pela ótica da sustentabilidade, para que não sejam os vetores de diversos impactos e os principais responsáveis pela geração de resíduos. Entretanto, mesmo com definição dada pela ONU sobre desenvolvimento sustentável, o termo sustentabilidade é muito subjetivo, e pode ser interpretado de diversas formas, o que dificulta o desenvolvimento de metodologias capazes de medir, quantificar e definir os indicadores relevantes para a análise.

Para realizar análises em produtos e serviços, há uma metodologia bastante difundida e utilizada no Brasil e no mundo todo, chamada de Avaliação do Ciclo de Vida (ACV), que analisa e compara produtos e serviços de forma holística, a partir do ponto de vista ambiental (ASSOCIAÇÃO BRASILEIRA DE NORMAS TÉCNICAS - ABNT, 2009a). Porém, a ideia de integrar os pilares econômicos e sociais na ACV já foi discutida por diversos autores (O'BRIEN; DOIG; CLIFT, 1996; NORRIS, 2001). Uma solução para complementar os métodos de avaliação de performance seria realizar a separação do termo sustentabilidade, muito abrangente, em três vertentes: social, ambiental e econômica. Dessa forma, pode-se analisar os três aspectos de forma detalhada, cada qual com metodologias específicas.

O aspecto ambiental pode ser avaliado pela ACV, que é um método já estabelecido, capaz de analisar diversas categorias de impacto ambiental, além de facilitar a identificação de processos ou fases operacionais em que os impactos ambientais sejam mais severos, sendo uma excelente ferramenta para auxiliar tomadas de decisões de empresas e partes interessadas (INSTITUTO BRASILEIRO DE INFORMAÇÃO EM CIÊNCIA E TECNOLOGIA - IBICT, 2019). A ACV pode ser utilizada para planejamento estratégico de organizações e indústrias, para selecionar indicadores e técnicas de medição de impactos ambientais mais eficientes, e auxiliar no marketing de, por exemplo, uma decisão política de grande impacto (ABNT, 2009a). Além disso, essa avaliação pode ser utilizada para auxiliar a introdução ou forçar a exclusão de produtos do mercado (GUINÉE; 


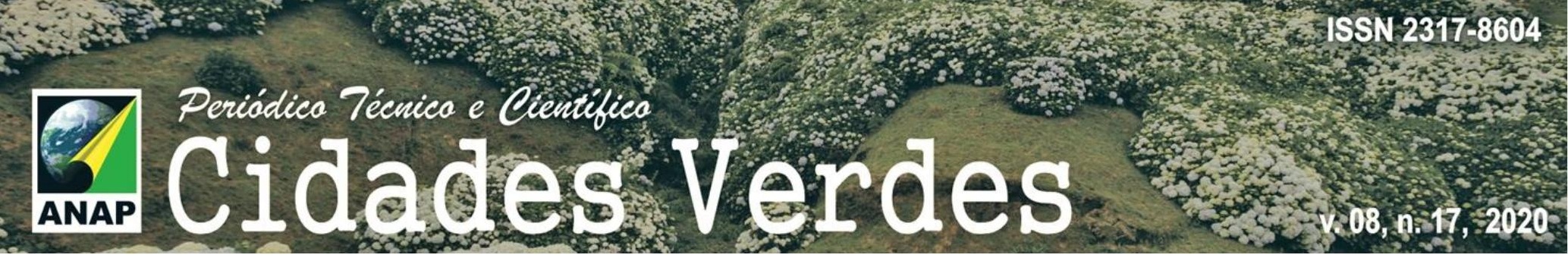

HAES; HUPPES, 1993). A ACV é normatizada pela série de normas ISO 14040, que estabelecem os princípios, a estrutura e os requisitos de uma ACV, e apresentam alguns exemplos de como aplicar essa análise (SOARES; SOUZA; PEREIRA, 2006). De acordo com a NBR ISO 14044 (ABNT, 2009b), os estudos de ACV devem incluir quatro etapas: definição de objetivo e escopo, análise de inventário do ciclo de vida (ICV), avaliação de impacto do ciclo de vida (AICV) e interpretação do ciclo de vida.

A vertente econômica da sustentabilidade possui uma metodologia de análise chamada de Avaliação de Custo do Ciclo de Vida, ou ACCV (LCC - Life Cycle Costing), que também segue o ciclo de vida dos produtos e serviços, analisando os custos incluídos nas fronteiras do estudo em questão (STAMFORD, 2020). A metodologia apresentada por Swarr et al. (2011) propõe que o Custo do Ciclo de Vida seria a soma dos diversos custos econômicos incorridos diretamente pelos agentes responsáveis pelo produto ou serviço. Entre esses custos, podemos citar: custo de capital; custos operacionais fixos e variáveis; custos com transporte entre as diversas etapas da produção; e os custos de gestão de resíduos, reciclagem e demolição.

Já as metodologias para avaliar o pilar social são as menos desenvolvidas. O Programa das Nações Unidas para o Meio Ambiente (PNUMA) já publicou orientações para a realização de Avaliações do Ciclo de Vida sociais (UNEP, 2009), mas a maioria das questões sociais relevantes são muito difíceis de medir (STAMFORD, 2020). Alguns exemplos para demonstrar a complexidade da situação seriam: como quantificar e decidir os indicadores gerais para medir a felicidade de uma população, ou então qual a definição exata de uma vida "saudável"? Por outro lado, muitas das métricas ambientais e econômicas já são apresentadas em formatos numéricos, facilitando sua quantificação. Alguns trabalhos, como os desenvolvidos por Kühnen e Hahn (2017) e Rafiaani et al. (2018) discutem essas questões e as dificuldades envolvidas. Entre os indicadores que podem ser utilizados nesse tipo de análise, estão: igualdade de gênero; empregabilidade; fatalidades devido a grandes acidentes; aumento do trânsito; proporção de investimentos em uma comunidade em relação aos lucros; entre outros.

A metodologia que integra os três pilares da sustentabilidade é chamada de Avaliação de Sustentabilidade do Ciclo de Vida (ASCV), e tem sido alvo de interesse de pesquisadores nos últimos anos (HUNKELER, 2006; KLÖPFFER, 2003; REBITZER; HUNKELER, 2003; WEIDEMA, 2005). A primeira vez que esse termo foi utilizado foi no estudo de Zhou, Jiang e Qin (2007), e com o desenvolvimento dessa metodologia, surgiram diversos questionamentos ainda sem resolução, sobre como aplicar ou como unificar diversas ferramentas diferentes em um único método (ZAMAGNI, 2012). Além disso, também há duas abordagens, a saber: A ASCV como a realização em conjunto da ACV, ACCV e da ACV social, sem nenhum tipo de ponderação; e a ASCV como sendo uma nova análise, utilizando o mesmo inventário da ACV, ou seja, incorporando os aspectos sociais, econômicos e categorias de impacto adicionais na etapa de Avaliação dos Impactos do Ciclo de Vida. Com esse trabalho, objetiva-se apresentar essa metodologia desenvolvida recentemente, explicitando suas vantagens e dificuldades, podendo servir como uma base de referência para novos trabalhos que sejam desenvolvidos utilizando a ASCV.

\section{OBJETIVOS}




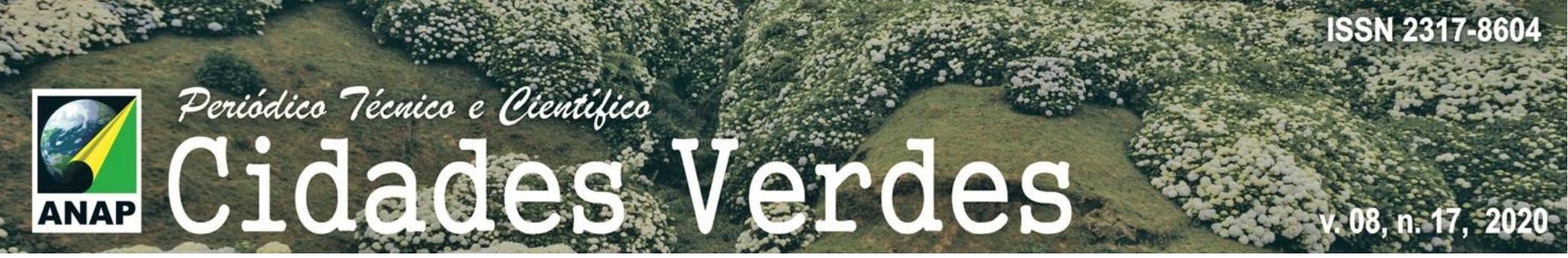

Reunir informações sobre a Avaliação de Sustentabilidade do Ciclo de Vida (ASCV), através de uma revisão bibliográfica, evidenciando seus atrativos, os desafios e os principais métodos utilizados, a fim de criar bases para tornar a ASCV uma ferramenta de apoio à sustentabilidade ambiental no Brasil, intensificando seu uso como método de avaliação de desempenho de produtos e serviços, com foco na área de construção civil.

\section{METODOLOGIA}

O procedimento metodológico utilizado foi uma revisão bibliográfica, em alguns dos principais periódicos científicos internacionais, utilizando a plataforma ScienceDirect, buscando informações sobre o tema, bem como as aplicações usuais, limitações e recomendações.

Para a pesquisa de artigos, foram utilizadas as seguintes palavras-chave: Life Cycle Sustainability Assessment, LCSA, Performance Assessment Methods e Civil Construction.

\section{RESULTADOS}

Tendo em vista que as particularidades de diferentes regiões de estudo podem influenciar as escolhas de métodos de análise, estão apresentados no Quadro 1 seis estudos encontrados, principalmente na área de construção civil, que utilizaram métodos de avaliação de performance, seus respectivos países, autores e ano de publicação.

Quadro 1: Métodos utilizados nos estudos, de acordo com o país de origem.

\begin{tabular}{|c|c|c|c|}
\hline Referência & Título do artigo & País & Métodos utilizados \\
\hline $\begin{array}{l}\text { Traverso et } \\
\text { al. (2012) }\end{array}$ & $\begin{array}{l}\text { Towards life cycle sustainability assessment: an } \\
\text { implementation to photovoltaic modules }\end{array}$ & $\begin{array}{l}\text { Alemanha } \\
\text { e Itália }\end{array}$ & $\begin{array}{l}A S C V=A C V+A C C V+A C V \\
\text { Social }\end{array}$ \\
\hline $\begin{array}{l}\text { Mateus e } \\
\text { Bragança } \\
(2018)\end{array}$ & $\begin{array}{l}\text { Avaliação da sustentabilidade da construção: } \\
\text { Desenvolvimento de uma metodologia para a } \\
\text { avaliação da sustentabilidade de soluções } \\
\text { construtivas }\end{array}$ & Portugal & $\begin{array}{l}\text { Metodologia de Avaliação } \\
\text { Relativa da } \\
\text { Sustentabilidade de } \\
\text { Soluções Construtivas } \\
\text { (MARS-SC). }\end{array}$ \\
\hline $\begin{array}{l}\text { Ekener et al. } \\
\quad \text { (2018) }\end{array}$ & $\begin{array}{c}\text { Developing Life Cycle Sustainability Assessment } \\
\text { methodology by applying values-based sustainability } \\
\text { weighting - Tested on biomass based and fossil } \\
\text { transportation fuels }\end{array}$ & Suécia & $\begin{array}{c}A S C V=A C V+A C C V+A C V \\
\text { Social }\end{array}$ \\
\hline $\begin{array}{l}\text { Silva et al. } \\
\text { (2018) }\end{array}$ & $\begin{array}{c}\text { Environmental, economic and social analysis of a } \\
\text { new cement composite produced with high } \\
\text { percentage of treated coconut }\end{array}$ & Brasil & ACV e AMBITEC/UESC \\
\hline $\begin{array}{l}\text { Gwerder et } \\
\text { al. (2019) }\end{array}$ & $\begin{array}{l}\text { Life beyond the grid: A Life-Cycle Sustainability } \\
\text { Assessment of household energy needs }\end{array}$ & Portugal & $\begin{array}{l}\text { ASCV e Análise de decisão } \\
\text { multicritério (ADMC) }\end{array}$ \\
\hline $\begin{array}{l}\text { Zheng et al. } \\
\text { (2019) }\end{array}$ & $\begin{array}{c}\text { Life-cycle sustainability assessment of pavement } \\
\text { maintenance alternatives: Methodology and case } \\
\text { study }\end{array}$ & China & $\begin{array}{c}A S C V=A C V+A C C V+A C V \\
\text { Social }\end{array}$ \\
\hline
\end{tabular}

Fonte: Elaborado pelo autor.

Em seguida, levantaram-se os resultados de cada estudo, em relação ao produto ou serviço avaliado, sob as perspectivas econômicas, ambientais e sociais. Estas informações estão apresentadas no Quadro 2. 


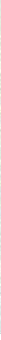

\begin{tabular}{|c|c|}
\hline Referência & Resultados \\
\hline $\begin{array}{l}\text { Traverso et } \\
\text { al. (2012) }\end{array}$ & $\begin{array}{l}\text { No quesito ambiental (ACV), o módulo fotovoltaico alemão, possui valores mais baixos de uso da } \\
\text { terra, frações potenciais desaparecidas e acidificação que o módulo italiano, tem maior potencial } \\
\text { de aquecimento global Os custos (ACCV) do módulo alemão são mais baixos que o italiano, (0,12 } \\
€ / m 2 \text { contra } 0,85 € / m 2 \text { ). Nos impactos sociais (ACV Social), os módulos alemães de } 2008 \text { e } 2009 \\
\text { se alternam entre melhor e o pior desempenho. Os resultados agregados, portanto, demonstram } \\
\text { que o modelo alemão de } 2008 \text { tem o melhor desempenho geral de sustentabilidade }\end{array}$ \\
\hline $\begin{array}{l}\text { Mateus e } \\
\text { Bragança } \\
(2018)\end{array}$ & $\begin{array}{l}\text { Mesmo não sendo a melhor solução nos aspectos técnicos e ambientais, no contexto geral, a } \\
\text { solução mais sustentável é a parede simples com reboco armado sobre isolante contínuo pelo } \\
\text { exterior, o que mostra a importância de uma análise com várias perspectivas. }\end{array}$ \\
\hline $\begin{array}{l}\text { Ekener et } \\
\text { al. (2018) }\end{array}$ & $\begin{array}{l}\text { O resultado classificando os tipos de combustível para transporte com base no desempenho da } \\
\text { sustentabilidade difere ao aplicar diferentes perfis de tomadores de decisão. Esse resultado } \\
\text { destaca que não há uma resposta única sobre qual das alternativas é mais sustentável, por } \\
\text { depender fortemente da visão de mundo e dos valores do tomador de decisão ou partes } \\
\text { interessadas. }\end{array}$ \\
\hline $\begin{array}{l}\text { Silva et al. } \\
\quad \text { (2018) }\end{array}$ & $\begin{array}{l}\text { O compósito desenvolvido com fibra de coco tratada tem potencial de reduzir os impactos } \\
\text { ambientais e a quantidade de insumos utilizados na confecção de materiais, além de criar } \\
\text { empregos e gerar uma economia de até } 21 \% \text {, quando comparado com o compósito convencional. } \\
\text { Com isso, seria possível aprimorar o desenvolvimento da construção brasileira de forma mais } \\
\text { sustentável. }\end{array}$ \\
\hline $\begin{array}{c}\text { Gwerder et } \\
\text { al. (2019) }\end{array}$ & $\begin{array}{l}\text { Atender às necessidades de energia das famílias de maneira sustentável requer uma análise do } \\
\text { contexto local e dos recursos disponíveis. Para residências remotas, as soluções de energia } \\
\text { renovável fora da rede fornecem uma forma confiável e sustentável de eletricidade e } \\
\text { aquecimento, assumindo que os proprietários tenham capital inicial para investir em tais } \\
\text { sistemas. As casas próximas à rede devem avaliar as vantagens e desvantagens entre sair da rede } \\
\text { e o potencial de conectar seus sistemas de energia renovável à rede para vender excesso de } \\
\text { geração. Para grupos de famílias próximas, o compartilhamento de sistemas de energia } \\
\text { renovável, solar ou eólico, por exemplo, é uma opção interessante a ser considerada para evitar } \\
\text { o superdimensionamento de sistemas individuais e permitir que estes sejam usados de forma } \\
\text { mais eficiente }\end{array}$ \\
\hline $\begin{array}{c}\text { Zheng et al. } \\
\text { (2019) }\end{array}$ & $\begin{array}{l}\text { O asfalto de mistura quente com asfalto recuperado (AMQR) foi a melhor alternativa nos aspectos } \\
\text { econômico e social, enquanto o asfalto de mistura quente com aditivo Sasobit de mistura quente } \\
\text { (AMQQ) alcançou o melhor desempenho ambiental. Na análise combinada, a solução AMQQ foi } \\
\text { a alternativa mais sustentável. }\end{array}$ \\
\hline
\end{tabular}

Fonte: Elaborado pelo autor.

Por fim, foi realizado o levantamento, em função do produto ou do serviço, das limitações, desafios, recomendações, orientações e soluções de cada um dos artigos revisados. As informações encontradas estão apresentadas no Quadro 3 
Quadro 3: Limitações, desafios e soluções de cada estudo.

\begin{tabular}{|c|c|c|c|}
\hline Referência & $\begin{array}{l}\text { Produto ou serviço } \\
\text { avaliado }\end{array}$ & Limitações e desafios & Recomendações e conclusões \\
\hline $\begin{array}{l}\text { Traverso } \\
\text { et al. } \\
(2012)\end{array}$ & $\begin{array}{l}\text { Produção de módulos } \\
\text { fotovoltaicos }\end{array}$ & $\begin{array}{l}\text { Ainda há dificuldades para uma } \\
\text { aplicação significativa da } \\
\text { metodologia ASCV, particularmente } \\
\text { as questões da seleção de } \\
\text { indicadores sociais e como ponderar } \\
\text { as diversas vertentes envolvidas. }\end{array}$ & $\begin{array}{l}\text { A metodologia de ASCV parece } \\
\text { ser uma boa ferramenta para } \\
\text { apoiar as tomadas de decisão } \\
\text { que consideram produção e } \\
\text { consumo sustentáveis. }\end{array}$ \\
\hline $\begin{array}{c}\text { Mateus e } \\
\text { Bragança } \\
\text { (2018) }\end{array}$ & $\begin{array}{l}\text { Soluções construtivas } \\
\text { convencionais e não } \\
\text { convencionais para } \\
\text { paredes exteriores } \\
\text { construtivas }\end{array}$ & $\begin{array}{l}\text { Os resultados da metodologia MARS- } \\
\text { SC estão intimamente ligados ao tipo } \\
\text { e número de parâmetros avaliados } \\
\text { dentro de cada indicador, e é } \\
\text { bastante dependente da ponderação } \\
\text { de cada um deles. }\end{array}$ & $\begin{array}{l}\text { Devem ser selecionados } \\
\text { tecnologias e materiais que } \\
\text { promovam a sustentabilidade no } \\
\text { domínio da construção. } \\
\text { O desenvolvimento e aplicação } \\
\text { de métodos de avaliação da } \\
\text { sustentabilidade são } \\
\text { fundamentais para as tomadas } \\
\text { de decisão que busquem } \\
\text { edifícios mais sustentáveis. }\end{array}$ \\
\hline $\begin{array}{l}\text { Ekener et } \\
\text { al. (2018) }\end{array}$ & $\begin{array}{l}\text { Etanol produzido a } \\
\text { partir da cana-de- } \\
\text { açúcar brasileira e } \\
\text { milho dos EUA, e } \\
\text { gasolina produzida a } \\
\text { partir de petróleo } \\
\text { russo e nigeriano }\end{array}$ & $\begin{array}{l}\text { Os resultados dependem muito das } \\
\text { partes envolvidas no estudo, suas } \\
\text { visões de mundo e prioridades As } \\
\text { principais divergências ocorrem nos } \\
\text { contextos sociais. }\end{array}$ & $\begin{array}{l}\text { A metodologia LCSA ainda } \\
\text { enfrenta desafios em relação à } \\
\text { integração de resultados, mas a } \\
\text { ADMC em conjunto com mais } \\
\text { informações sobre as partes } \\
\text { interessadas, parece ser uma } \\
\text { abordagem útil para aprimorar } \\
\text { ainda as avaliações futuras. }\end{array}$ \\
\hline $\begin{array}{l}\text { Silva et al. } \\
\quad(2018)\end{array}$ & $\begin{array}{l}\text { Compósito cimentício } \\
\text { produzido com } \\
\text { elevado teor fibra de } \\
\text { coco tratada }\end{array}$ & $\begin{array}{l}\text { A metodologia original } \\
\text { (AMBITEC/EMBRAPA) não retratava } \\
\text { de forma adequada o compósito em } \\
\text { estudo, sendo necessário realizar } \\
\text { modificações para que o método } \\
\text { fosse utilizado (passando a se } \\
\text { chamar AMBITEC/UESC). }\end{array}$ & $\begin{array}{l}\text { Os métodos empregados no } \\
\text { estudo } \\
\text { convergência } \\
\text { indicadores ambientraram } \\
\text { indar os } \\
\text { integram a AMBITEC/UESC com } \\
\text { categorias de impacto da ACV. }\end{array}$ \\
\hline $\begin{array}{l}\text { Gwerder } \\
\text { et al. } \\
\text { (2019) }\end{array}$ & $\begin{array}{lr}\text { Necessidades } & \text { de } \\
\text { eletricidade } & \mathrm{e} \\
\text { aquecimento } & \text { em } \\
\text { casas fora da } & \text { rede } \\
\text { elétrica } & \\
\end{array}$ & $\begin{array}{l}\text { Os resultados da ASCV são } \\
\text { fortemente influenciados pelos } \\
\text { indicadores selecionados e pelas } \\
\text { necessidades e tecnologias dos } \\
\text { moradores das casas. }\end{array}$ & $\begin{array}{l}\text { A combinação da ASCV e ADMC é } \\
\text { útil para avaliar os impactos da } \\
\text { sustentabilidade, incorporando } \\
\text { as preferências dos tomadores } \\
\text { de decisão. }\end{array}$ \\
\hline $\begin{array}{l}\text { Zheng et } \\
\text { al. (2019) }\end{array}$ & $\begin{array}{l}\text { Alternativas } \\
\text { manutenção } \\
\text { pavimentos }\end{array}$ & $\begin{array}{l}\text { São necessários trabalhos futuros } \\
\text { para complementar e aperfeiçoar a } \\
\text { metodologia, como a realização de } \\
\text { uma análise da incerteza e a } \\
\text { formação de um painel internacional } \\
\text { e multidisciplinar de especialistas. }\end{array}$ & $\begin{array}{l}\text { O estudo busca auxiliar na } \\
\text { seleção da alternativa de } \\
\text { pavimento mais apropriada. No } \\
\text { entanto, a metodologia proposta } \\
\text { pode não representar totalmente } \\
\text { a sustentabilidade do pavimento, } \\
\text { podendo ser incorporados novos } \\
\text { bancos de dados e informações }\end{array}$ \\
\hline
\end{tabular}

Fonte: Elaborado pelo autor.

Observou-se que foram encontrados poucos estudos que realizaram análises de sustentabilidade com a temática de construção civil, sendo levantados alguns estudos com foco em questões energéticas, e que algumas outras metodologias também foram utilizadas, mas a maioria dos artigos encontrados emprega a metodologia ASCV. Isso demonstra que esse tipo de análise está em ascensão, corroborando com o estudo de Costa, Quinteiro e Dias (2019), que realizaram uma revisão sistemática da $\mathrm{ASCV}$, concluindo que há uma onda crescente no número de artigos sobre 


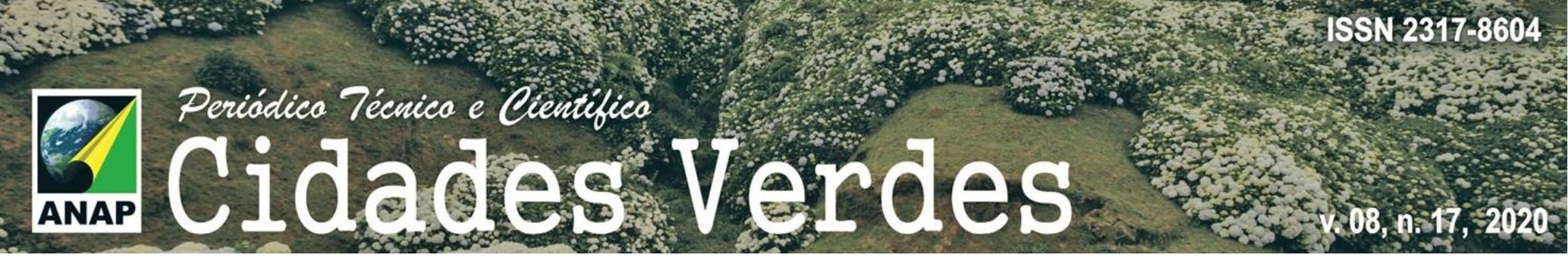

o tema, com países de origem variados. Outro ponto em comum com o resultado desse artigo é que o método mais utilizado para operacionalizar e integrar as diferentes metodologias envolvidas na ASCV é a Análise de Decisão Multicritério, por ser amplamente difundido.

Além disso, uma limitação bastante recorrente nos estudos foi a relevância das escolhas e preferências das partes interessadas e tomadores de decisão. Nesse ponto, o método em questão se aproxima bastante da $\mathrm{ACV}$, já que também foi desenvolvido seguindo o escopo da ISO 14040. Porém, verifica-se que é necessário, agora que a fase de surgimento e criação da metodologia já ocorreu, elaborar um procedimento normatizado de como realizar a ASCV, para que os estudos sejam realizados com metodologias similares e comparáveis.

Outro fato interessante é a rapidez do Brasil para incorporar esse novo tipo de análise em seu repertório, ao lançar no dia 23 de maio de 2019 o Programa de Avaliação da Sustentabilidade do Ciclo de Vida (BioCycleS) de Produtos, através do IBICT (Instituto Brasileiro de Informação em Ciência e Tecnologia). Esse programa tem como algumas de suas metas: apoiar a comunidade acadêmica, oferecendo disciplinas sobre o tema para cursos de graduação e pós-graduação; incentivar a construção e a constante melhoria das bases de dados nacionais; aprimorar metodologias que avaliem os impactos de sistemas de produtos; e incentivar a formação de parcerias público privadas para ampliar a aplicação de estudos de avaliação nos variados sistemas produtivos e produtos nacionais (IBICT, 2019).

\section{CONCLUSÃO}

Pode-se concluir, portanto, que uma das maiores dificuldades de implementar amplamente a ASCV é realizar a integração harmoniosa entre os vários métodos componentes. Para resolver essa questão, é necessário que os objetivos do estudo, da mesma forma que em uma ACV, sejam claros, as fronteiras do sistema sejam bem definidas, e os bancos de dados abastecidos com mais dados sociais e econômicos, permitindo assim uma melhor escolha de categorias de impacto relevantes para a situação.

Outro ponto a ser resolvido é a falta de interesse de compradores e vendedores, ou, no caso da construção civil, dos clientes e das construtoras, em incorporar sistemas e materiais que incorporem a sustentabilidade desde a etapa de idealização até a execução. Para tentar amenizar esse problema, sugere-se desenvolver as formas e estratégias de disseminação dos resultados de estudos com essa temática, para que os benefícios possam ser entendidos e adotados pelas partes interessadas. Outra ação possível é incorporar em manuais e normas algumas exigências mínimas de sustentabilidade nas construções, forçando uma maior aderência inicial das partes envolvidas, mas para que posteriormente, o fator sustentabilidade seja considerado um diferencial na venda dessas construções, sejam elas totalmente novas ou apenas melhorias dos sistemas já existentes.

A normatização dessa metodologia também resolveria outra questão, que é a dificuldade de se comparar os estudos de avaliação de sustentabilidade, pois estes utilizam categorias de impacto e métodos variados ou adaptados. Com uma maior uniformidade, assim como ocorreu com a ACV ao se criar a ISO 14040, a comparação entre os estudos, o desenvolvimento e o aprimoramento da ASCV será bastante facilitado, dando margem para a ampla incorporação desse método em avaliações realizadas pelo mundo todo. Por fim, ainda é necessária a 


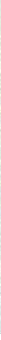

realização de mais estudos de caso com esse tipo de avaliação, em setores e áreas de diferentes conhecimentos, para que as particularidades de cada um se evidenciem e possam ser resolvidas da forma mais harmoniosa possível.

\section{AGRADECIMENTO}

Os Autores gostariam de agradecer a Fundação de Amparo à Pesquisa do Estado de São Paulo (FAPESP) e a Universidade Estadual Paulista "Júlio de Mesquita Filho" (UNESP - Campus de Ilha Solteira) pelo apoio recebido.

\section{REFERÊNCIAS BIBLIOGRÁFICAS}

ASSOCIAÇÃO BRASILEIRA DE NORMAS TÉCNICAS. NBR ISO 14040: Gestão ambiental - Avaliação do ciclo de vida Princípios e estrutura. Rio de Janeiro, 2009a.

ASSOCIAÇÃO BRASILEIRA DE NORMAS TÉCNICAS. NBR ISO 14044: Gestão ambiental - Avaliação do ciclo de vida Requisitos e orientações. Rio de Janeiro, 2009b.

AZEVEDO, J. L. A economia circular aplicada no Brasil: uma análise a partir dos instrumentos legais existentes para a logística reversa. In: XI Congresso Nacional de Excelência em Gestão, Rio de Janeiro. Anais... Rio de Janeiro: NITS, 2015. p. 1-16.

BATLOUNI NETO, J. Critérios de projetos para seleção de materiais. In: ISAIA, G. C. Materiais de construção civil e princípios de ciência e engenharia de materiais. São Paulo: IBRACON, 2007. p.1792.

COSTA, D.; QUINTEIRO, P.; DIAS, A.C. A systematic review of life cycle sustainability assessment: Current state, methodological challenges, and implementation issues. Science Of The Total Environment, v. 686, p.774-787, out. 2019.

GUINÉE, J. B.; HAES, H. A. U.; HUPPES, G. Quantitative life cycle assessment of products: goal definition and inventory. Journal of Cleaner Production, v.1, n.1, p. 3-13. 1993.

GWERDER, Y. V.; MARQUES, P.; DIAS, L. C.; FREIRE, F. Life beyond the grid: A Life-Cycle Sustainability Assessment of household energy needs. Applied Energy, v. 255, dez. 2019.

HUNKELER, D. Societal LCA Methodology and Case Study. The International Journal Of Life Cycle Assessment, v. 11, n. 6, p.371-382, 7 ago. 2006.

INSTITUTO BRASILEIRO DE INFORMAÇÃO EM CIÊNCIA E TECNOLOGIA - IBICT. Avaliação do Ciclo de Vida. Disponível em: <http://acv.ibict.br/>. Acesso em: 17 OUT. 2019.

KLÖPFFER, W. Life-Cycle based methods for sustainable product development. The International Journal Of Life Cycle Assessment, v. 8, n. 3, p.157-159, mai. 2003.

KÜHNEN, M.; HAHN, R. Indicators in Social Life Cycle Assessment: A Review of Frameworks, Theories, and Empirical Experience. Journal Of Industrial Ecology, v. 21, n. 6, p.1547-1565, set. 2017.

LOBO, L. Como construir de forma sustentável e economizar? 2016. Disponível em: <http://www.barearquitetura.com/como-construir-de-forma-sustentavel-e-economizar/>. Acesso em: 25 out. 2019.

MARCELINO-SADABA, S.; KINUTHIA, J.; OTI, J.; SECO MENESES, A. Challenges in Life Cycle Assessment (LCA) of stabilised clay-based construction materials. Applied Clay Science, v. 144, p.121-130, ago. 2017.

MATHEUS, R.; BRAGANÇA, L. Avaliação da sustentabilidade da construção: Desenvolvimento de uma metodologia para a avaliação da sustentabilidade de soluções construtivas. In: Congresso sobre Construção Sustentável, 1., 2004, Leça de Palmeira, Portugal. Anais. Leça de Palmeira: Ordem dos Engenheiros, 2004. 


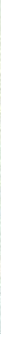

MEDEIROS, L. M.; DURANTE, L. C.; CALLEJAS, I. J. A. Contribuição para a avaliação de ciclo de vida na quantificação de impactos ambientais de sistemas construtivos. Ambiente Construído, Porto Alegre, v. 18, n. 2, p. 365-385, abr. 2018.

NAÇÕES UNIDAS. Relatório da Comissão Mundial sobre o Meio Ambiente e Desenvolvimento, 42/187, 1987.

NORRIS, G. A. Integrating life cycle cost analysis and LCA. The International Journal Of Life Cycle Assessment, v. 6, n. 2, p.118-120, mar. 2011.

O'BRIEN, M.; DOIG, A.; CLIFT, R. Social and environmental life cycle assessment (SELCA). The International Journal Of Life Cycle Assessment, v. 1, n. 4, p.231-237, dez. 1996.

RAFIAANI, P.; KUPPENS, T.; DAEL, M. V.; AZADI, H.; LEBAILLY, P.; PASSEL, S. V. Social sustainability assessments in the biobased economy: Towards a systemic approach. Renewable And Sustainable Energy Reviews, v. 82, p.1839-1853, fev. 2018.

REBITZER, G.; HUNKELER, D. Life cycle costing in LCM: ambitions, opportunities, and limitations. The International Journal Of Life Cycle Assessment, v. 8, n. 5, p.253-256, set. 2003.

ROODMAN, D. M.; LENSSEN, N. K.; PETERSON, J. A. A building revolution: how ecology and health concerns are transforming construction. Washington, DC: World Watch Paper No. 124, World Watch Institute. 1995.

SILVA, E. J.; VELASCO, F. C. G.; LUZARDO, F. H. M.; MARANDUBA, H. L.; MARQUES, M. L. Avaliação ambiental, econômica e social de um novo compósito cimentício produzido com elevado teor fibra de coco tratada. Revista Ibero-americana de Ciências Ambientais, v. 9, n. 4, p.253-267, 23 mai. 2018.

SOARES, S. R.; SOUZA, D. M.; PEREIRA, S. W. A avaliação do ciclo de vida no contexto da construção civil. In: SATTLER, M. A.; PEREIRA, F. O. R. (Ed.). Construção e Meio Ambiente. Porto Alegre: ANTAC, 2006. Cap. 4. p. 96-127.

STAMFORD, L. Life cycle sustainability assessment in the energy sector. Biofuels For A More Sustainable Future, p.115-163, 2020

SWARR, T. E.; HUNKELER, D.; KLÖPFFER, W.; PESONEN, H.-L.; CIROTH, A.; BRENT, A. C.; PAGAN, R. Environmental life-cycle costing: a code of practice. The International Journal Of Life Cycle Assessment, v. 16, n. 5, p.389-391, 27 abr. 2011.

TAVARES, S. F. Metodologia de Análise do Ciclo de Vida Energético de Edificações Residenciais Brasileiras. 2006. 225 f. Tese (Doutorado) - Escola de Engenharia, Universidade Federal de Santa Catarina, Florianópolis, 2006.

TEIXEIRA, L. P.; CARVALHO, F. M. A. A construção civil como instrumento de desenvolvimento da economia brasileira. Revista paranaense de desenvolvimento, Curitiba, n.109, p.09-26, 2005.

TRAVERSO, M.; ASDRUBALI, F.; FRANCIA, A.; FINKBEINER, M. Towards life cycle sustainability assessment: an implementation to photovoltaic modules. The International Journal Of Life Cycle Assessment, v. 17, n. 8, p.10681079, 16 mai. 2012.

UNIÃO EUROPEIA (UE), Comissão das Comunidades Europeias. Comunicação da Comissão Europeia ao Conselho, ao Parlamento Europeu, ao Comitê Econômico e Social Europeu e ao Comitê das Regiões: Para uma estratégia temática sobre o Ambiente Urbano. COM (2004) 60, Bruxelas, Bélgica, 2004.

UNITED NATIONS ENVIRONMENT PROGRAMME - UNEP. Guidelines for Social Life Cycle Assessment of a Product. Bélgica, 2009.

WEIDEMA, B. P. The Integration of Economic and Social Aspects in Life Cycle Impact Assessment. The International Journal Of Life Cycle Assessment, v. 11, n. 1, p.89-96, 6 dez. 2005.

YUSOF, N.; IRANMANESH, M. The Impacts of Environmental Practice Characteristics on Its Implementation in Construction Project. Procedia Environmental Sciences, v 37, p. 549-555, 2017. 


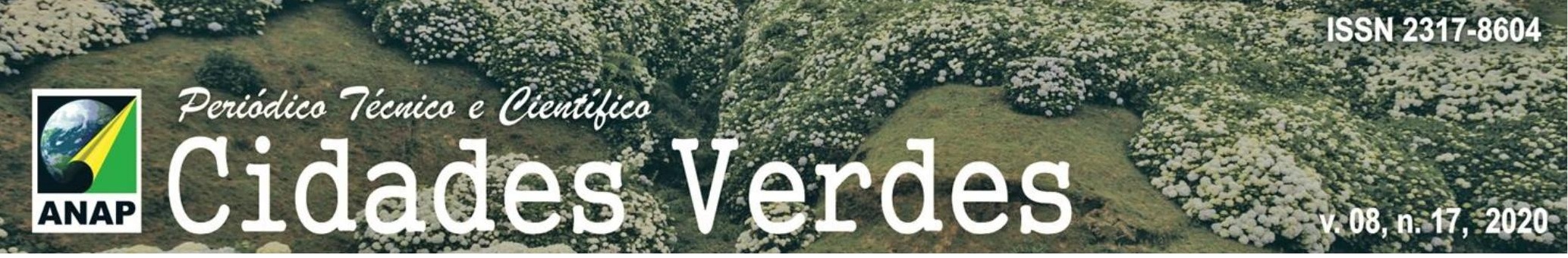

ZAMAGNI, A. Life cycle sustainability assessment. The International Journal Of Life Cycle Assessment, v. 17, n. 4, p.373-376, 22 fev. 2012.

ZHENG, X.; EASA, S. M.; YANG, Z.; JI, T.; JIANG; Z. Life-cycle sustainability assessment of pavement maintenance alternatives: Methodology and case study. Journal Of Cleaner Production, v. 213, p.659-672, mar. 2019.

ZHOU, Z; JIANG, H.; QIN, L. Life cycle sustainability assessment of fuels. Fuel, v. 86, n. 1-2, p.256-263, jan. 2007. 